\title{
Ureteral stone extraction under direct ureteroscopic vision: An alternative for routine ureteral stone management
}

\author{
Hassan Niroomand ${ }^{1 *}$, Sima Binaafar ${ }^{1}$, Amir Ehsan Shayegan ${ }^{1}$, Mohsen Varyani ${ }^{1}$, Bijan \\ Rezakhaniha $^{1}$
}

1. Trauma Research Center, AJA University of Medical Sciences, Tehran, Iran

*Corresponding author:Tel: +98 9127160698 Fax: +98 2188337932

Address: Trauma Research Center, AJA University of Medical Sciences, Etemadzadeh St. Fatemi Ave.

Tehran. Iran

E-mail: hassanniroomand@ajaums.ac.ir

Received: 2018/05/31 revised: 2018/07/3 accepted: 2018/08/7

\begin{abstract}
Introduction: During recent years, considerable efforts have been expended into the management of urinary stone. Here, we present our experience on ureteric stone removal without any lithotripsy interventions. Combination direct vision with basket en-trapping provided a new dimension to our ureteroscope experience.

Materials and methods: Here, we reviewed the medical data of our adult patients with $\leq 10$ $\mathrm{mm}$ stone size, who received primary stone extraction under direct ureteroscopic vision without lithotripsy during a 2-year period. During the six months of follow-up every patient was seen frequently.

Results: The study included 69 patients from both sexes with ages ranging from 18 to 68 years. We obtained $92.7 \%$ success rate. The average length of operative procedures was 25.3 \pm 10.4 min with a $14.4 \%$ complication rate.

Conclusion: Ureteral stone extraction requires considerable caution and may be associated with some complications. Stone extraction under direct ureteroscope guidance facilitates this procedure, especially in the distal stones. It seems combination direct live imaging with basket en-trapping may be helpful in these precise cases.
\end{abstract}

Keywords: Urinary stone, Direct vision, Ureteroscopy, Basket en-trapping, Adult

\section{Introduction}

Urinary stone disease is a major health problem that affects $2-3 \%$ of the population. Today, several endourologic options, such as Ureteroscopic Lithotripsy, Shock Wave Lithotripsy (SWL), Laparoscopic Lithotomy, and Percutaneous Nephrolithotomy, are available for treating this problem (1-5). Each type of these treatments is associated with related benefits and risks (6-8). For example retropulsion during endoscopic lithotripsy occurs in 5 to 40 percent of the cases (4).

\begin{abstract}
Progress in ureteroscopic technology,specially advancesin small semi rigid and flexible ureteroscopes lead to prevent needless interventions and enable urologists to remove the stone. There are many varieties of tools that help better stone ureteroscopic removal such as baskets, forceps and graspers (9-10). The ureteroscopic removal of the stone with a basket is a mechanical approach that can perhaps be used instead of different lithotripsy techniques.
\end{abstract}

Copyright (C) 2018 Journal of Basic Research in Medical Science. This is an open access article distributed under the terms of the Creative Commons Attribution 4.0 International License (https://creativecommons.org/licenses/by/4.0/) which permits copy and redistribute the material, in any medium or format, provided the original work is properly cited. 
Here, we present our experience on ureteric stone removal without any lithotripsy interventions. We combined direct vision under ureteroscope with basket en- trapping. To our knowledge, this is a first report of ureteroscopic stone removal that just applying this mechanical approach under direct vision.

\section{Materials and methods}

The medical data of our patients, who received primary stone extraction under direct ureteroscopic vision without lithotripsy during a 2-year period, were reviewed. They were adult patients with $\leq 10 \mathrm{~mm}$ stone size without renal anatomical anomalies who did not suffer from all spectrums of urinary tract infection (UTI) (active/ chronic/ recurrent). Also, someone who had experienced any previous lithotripsy management was excluded. This study was reviewed and approved by the Research Committee of AJA University of Medical Sciences, Tehran, Iran (Code No. 84/91/408).

\section{Preoperative Evaluation: The} preoperative evaluation that included detailed history, physical examination, routine laboratory tests together with plain $\mathrm{X}$-ray of the kidneys, ureters and bladder in addition to excretory urography (IVP), ultrasound or computerized tomography (CT)was carried out in all patients.

The stone status and size were evaluated postoperatively. All of the patients also received the same prophylactic IV antibiotics.

Operative procedure: The operations were performed under general or spinal anesthesia in a lithotomic position regarding the vital functions' management. The procedure was performed by using a 7.5/8 Fr. ureteroscope. Then a safety guide wire $(0.038$ inch) was introduced to the ureter. Removing the stone was done by insertion extractor basket in to working channel under direct vision. Then stones can be entrapped in the basket. Synchronic pressurized washing liquid created a pressure mechanism that can be pushed the calculi. When the stone is trapped in a basket, it may be failing to pass through distal of the ureter. However, at this time, ureteroscopic manipulation with the upward movement may be introduced to obtain a relatively dilatation. In case of need, double J stent would be embedded post-operatively, remaining for up to two weeks. All patients were discharged on the first day after the operation without complications.

Inter/Postoperative evaluation: Also stone residual fragments and ureteral status were assessed at interoperation and then 3 to 6 months after discharge.

Follow up: All patients were examined at our out-patient clinic during regular visits for a six months follow up period.

Assessment of procedure outcome: The operative procedure was considered successful if complete ston e-free status was achieved and no particular complaints were mentioned by including all months of follow-up.

\section{Statistical analysis}

Using SPSS (v. 15.0), statistical analyses were performed and a value of $\mathrm{P}<0.05$ was considered significant. In summary, to categorize dependent on variables, we used the mean and standard deviation for Continuous variables and absolute and relative frequencies for categorical variables.

\section{Results}

Here, we report the results of ureteral stone extraction in 69 patients of both sexes who ranged from 18 to 68 years. Also, stone size ranged from 3-10 $\mathrm{mm}$ (Table 1).

Table 1: Brief report of study results

\begin{tabular}{|l|l|}
\hline Number of patients & 69 \\
\hline Gender (male/ female) & $48 / 21$ \\
\hline Ages (year) & $32.5 \pm 12^{*}$ \\
& Range (18-68) \\
\hline Stone location & Proximal: $11(16 \%)$ \\
& Middle: $19(27.5 \%)$ \\
& Distal: $39(56.5 \%)$ \\
\hline Stone size (mm) & $6.7 \pm 1.6^{*}$ \\
& Range (3-10) \\
\hline
\end{tabular}

* Data are shown mean \pm SD 
We perform basket stone extraction under direct ureteroscopic vision for 64 patients (64 from 69 patients). Stone was located in the proximal, middle and distal of ureter, respectively in $8(12.5 \%), 17(26.6 \%)$ and $39(60.9 \%)$ cases. Totally basketing under direct ureteroscopic vision without lithotripsy associated with a $14.4 \%$ complication: $4.7 \%(n=3)$ ureteral mucosa rupture during ureteroscopy and $10.9 \%$ $(n=7)$ post ureteroscopy .Also, ureteral mucosa rupture was managed by embedding double $\mathbf{J}$ stent. In all of them, the stent was removed within two weeks and IVP was performed. Also, ureteral constrictions and/or contrast extravasations was not observed in any of them. The post ureteroscopy complications included $2.9 \%$ $(\mathrm{n}=2)$ UTI and $7.2 \% \quad(\mathrm{n}=5)$ irrigative symptoms which were related to stent placement and improved after stent removal.

Also pneumatic lithotripsy was done for five patients $(n=5 / 69)$. In these patients, stone was located in the upper $(n=3)$ and middle of $(n=2)$ the ureter.

Study population at 6 months follow-up did not report any complications and no patients have attended another institution with pain, sepsis, etc.

For stones $\leq 5 \mathrm{~mm}$, the success rate was $98.5 \%$ and for stones $>5 \mathrm{~mm}$, this rate was $94.2 \%$. These results were obtained in 25.3 \pm 10.4 min operative time.

Also Figure 1 illustrates a stone that was entrapped in basket; we could monitor all of the procedure in this technique.

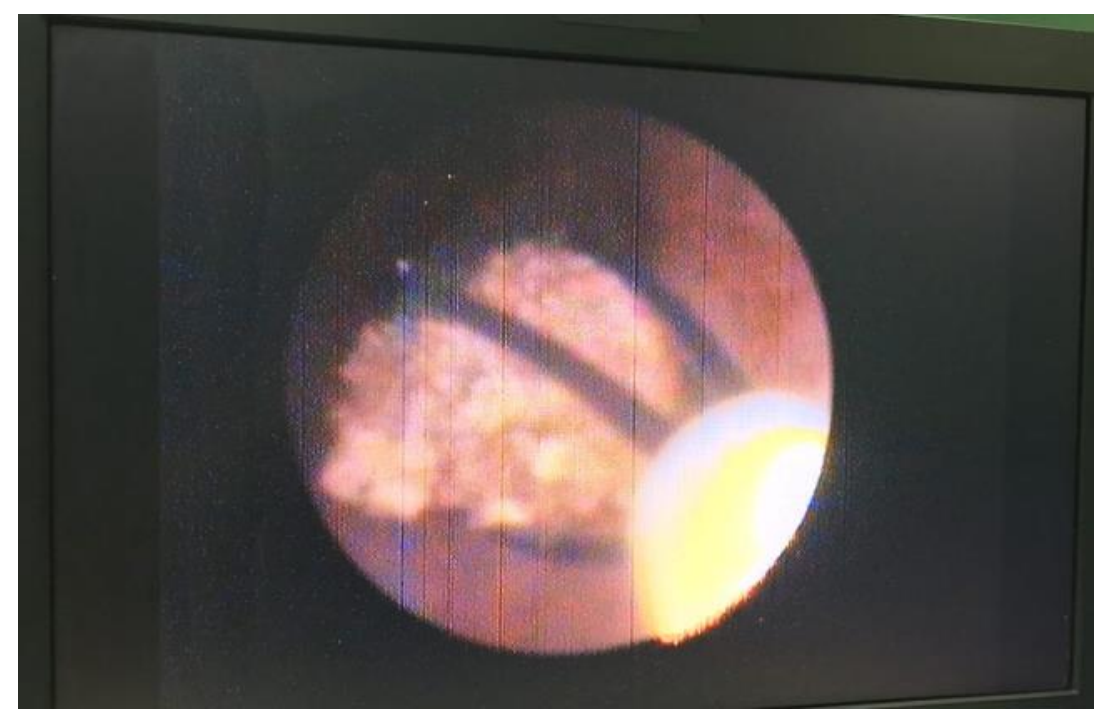

Figure1. Monitoring the procedure of stone entrapping in Basket. When the operation was performed in visual condition, the surgeons can follow the stone en-trapping easily. Also, when the stone is released, the surgeons know it immediately and can manage operating procedure.

\section{Discussion}

In light of the experienced surgeon, we obtained $92.7 \%$ successful rate. This finding is too good. Present result of stonefree rate is almost $11 \%$ higher than Castro et al. outcome. They used semirigid ureteroscopy for all stone locations and reported $83.8 \%$ stone-free rates. They have seen no significantly difference in stonefree rates between semirigid and flexible ureteroscopes procedures - Although, they analyzed large population who received ureteroscopy (9681 patients), but interestingly, they achieved different result from the 2007 American Urological Association (AUA) Guidelines on the Management of Ureteral Calculi data. Access to the proximal ureter is one of important factor to increase stone-free rates. In this regard using flexible ureteroscopy was suggested by AUA as a better option compared with rigid or 
semirigidureteroscopes because of a $10 \%$ more stone-free rates (11-12).

Another point of our approach to ureteral stone management was prevention of needless intervention or complications, which were related to lithotripsy. Lithotripsy techniques such as shock wave lithotripsy (SWL) or holmium-YAG laser lithotripsy, usually accompanied by significant risk of high complications such as ureteral perforation or stricture development or ureteral stricture rate or ureteral dilation. Moreover, in cases with high body mass index, SWL setting is difficult $(6,8)$. Although long-term practice decreases subsequent complications, but ureteroscopy help decrease inter lithotripsy traumas. Here we avoid any stone manipulation and stone just was en-trapped in the basket under direct ureteroscopy view. Karadag et al, analyzing the data of 124 patients, reported $63.4 \%$ and $86.8 \%$ successful rate for initial stone free status in groups 1 and 2, respectively. Their groups were defined as patients who were preformed semirigid (S-URS) or flexible ureterorenoscopic (F-URS) lithotripsy with holmium: YAG laser, respectively. The reoperation was required in $11.3 \%$ of cases which may arise from the mean stone size. The average stone size was $12.5 \mathrm{~mm}$ and reoperation was required in cases that had rest stones or stones $>4 \mathrm{~mm}$ in radiologic evaluations (9).

Although topic of stone free status is complex and influenced by some agents, there is also published treatment trials to guide the usefulness of $\mathrm{N}$-Trap ${ }^{\circledR}$ basket in combination with semirigid URS. These empirical literatures support the higher success rate achievement. For example Liu et al, reported significantly higher stonefree rate in group of patients who were treated by semi-rigid ureteroscope with the aid of stone basket $(n=135 ; 93.2 \%)$ compared with that of patients without the aid of the basket $(n=52 ; 51.6 \%)(13)$. In the present study we used only basket entrapping under direct ureteroscope vision for all of our patients $(n=64)$, this procedure accompanied with totally $100 \%$ successful rate without any lithotripsy requested. Wherever Liu and colleagues used holmium: YAG laser lithotripsy for their patients (13). Although, due to the risk of incidence of complications such as avulsion, pneumatic lithotripsy was used for five patients $(n=5 / 69,7.2 \%)$.

The next advantage is reduction operating time. In our series the operating time was $25.3 \quad \pm 10.4$ min while Karadag et al. have reported higher rate: $64.71 \pm 16.11 \mathrm{~min}$ for Semirigid URS and $84.06 \pm 16.7 \mathrm{~min}$ for Flexible URS (9). This outcome achieved under direct ureteroscope vision in a single - step procedure. Also, in this study, JJ stent was used for only three patients $(4.3 \%)$. Although there is an old view point about necessity of stent therapy as aroutine part of the postoperative care, but this was in agreement with Elashry et al.(14).

\section{Conclusion}

Combination basket en-trapping with direct vision under ureteroscope is generally looked on as being more satisfactory than the blind use of many varieties of tools such as baskets, forceps and graspers, because it avoids the related risks such as retropulsion. This approach forms the basis to creation of a safe model, one that contains direct vision in all of the ureteric stone removal.

\section{Acknowledgement}

We would like to thank all of the individuals who kindly accepted to participate in this study

\section{Conflict of interest}

The authors declare that they have no conflict of interest. 


\section{References}

1. Chotikawanich E, Korman E, Monga M. Complications of stone baskets: 14year review of the manufacturer and user facility device experience database. J Urol. 2011;185(1):179-83. doi: 10.1016/j.juro.2010.08.091.

2. Anagnostou T, Tolley D. Management of ureteric stones. Eur Urol. 2004;45(6):714-21. doi.org/10.1016/j.eururo.2003.10.018

3. Kiraç M, Atkin MS, Biri H, Deniz N. Ureteroscopy: the first-line treatment for distally located ureteral stones smaller than $10 \mathrm{~mm}$. Urol J. 2014;10(4):1028-34.

4. Molina WR, Pompeo A, Sehrt D, Pohlmann G, Kim FJ. [Use of a polymeric gel to prevent retropulsion during intracorporeal lithotripsy]. Actas Urol Esp. 2013;37(3):188-92. doi: 10.1016/j.acuro.2012.04.006. (Spanish)

5. Khoder WY, Bader M, Sroka R, Stief C, Waidelich R. Efficacy and safety of Ho: YAG laser lithotripsy for ureteroscopic removal of proximal and distal ureteral calculi. BMC Urol. 2014;14:62. doi: 10.1186/1471-2490-14-62.

6. Geavlete P, Georgescu D, NiţĂ G, Mirciulescu V, Cauni V. Complications of 2735 retrograde semirigidureteroscopy procedures: a single-center experience. J Endourol. 2006;20(3):179-85. doi.org/10.1089/end.2006.20.179

7. Oberlin DT, Flum AS, Bachrach L, Matulewicz RS, Flury SC. Contemporary surgical trends in the management of upper tract calculi. J Urol. 2015;193(3):880-4. doi: 10.1016/j.juro.2014.09.006.

8. Sancak EB, Akbas A. Treatment strategies for large impacted upper ureteral stones. Can Urol Assoc J. 2016;10(7-8):287.
9. Karadag MA, DemirA, Cecen K, Bagcioglu M, Kocaaslan R, Sofikerim $\mathrm{M}$, et al. Flexible ureterorenoscopy versus semirigidureteroscopy for the treatment of proximal ureteral stones: a retrospective comparative analysis of 124 patients. Urol J. 2014;11(5):186772. doi.org/10.22037/uj.v11i05.2513

10. Kroczak TJ, Kaler KS, Patel P, AlEssawi T. Ureteroscopy with conscious sedation for distal ureteric calculi: 10year experience. Can Urol Assoc J. 2016;10(1-2):E12-6. doi: 10.5489/cuaj.3302.

11. Castro EP, Osther PJ, Jinga V, Razvi H, Stravodimos KG, Parikh K et al. Differences in ureteroscopic stone treatment and outcomes for distal, mid, proximal, or multiple ureteral locations: the Clinical Research Office of the Endourological Society ureteroscopy global study. Eur Urol. 2014;66(1):102-9.

doi: 10.1016/j.eururo.2014.01.011.

12. Preminger GM, Tiselius HG, Assimos DG, Alken P, Buck C, Gallucci M et al. 2007 guideline for the management of ureteral calculi. J Urol. 2007;178(6):2418-34. doi.org/10.1016/j.juro.2007.09.107

13. Liu DY, He HC, Wang J, Tang Q, Zhou YF, Wang MW, et al. Ureteroscopic lithotripsy using holmium laser for 187 patients with proximal ureteral stones. Chin Med J (Engl). 2012;125(9):15426.

14. Elashry OM, Elgamasy AK, Sabaa MA, Abo-Elenien M, Omar MA, Eltatawy $\mathrm{HH}$, et al. Ureteroscopic management of lower ureteric calculi: a 15-year single-centre experience. BJU Int. 2008;102(8):1010-7. doi: 10.1111/j.1464-410X.2008.07747.x. 